\title{
Dynamic modulation of innate immunity programming and memory
}

\author{
Ruoxi Yuan \& Liwu Li ${ }^{*}$ \\ Department of Biological Science, Pathobiology and Biomedical Sciences, Virginia Tech, Blacksburg VA24061-0910, USA
}

Received December 7, 2015; accepted December 28, 2015; published online January 5, 2016

\begin{abstract}
Recent progress harkens back to the old theme of immune memory, except this time in the area of innate immunity, to which traditional paradigm only prescribes a rudimentary first-line defense function with no memory. However, both in vitro and in vivo studies reveal that innate leukocytes may adopt distinct activation states such as priming, tolerance, and exhaustion, depending upon the history of prior challenges. The dynamic programming and potential memory of innate leukocytes may have far-reaching consequences in health and disease. This review aims to provide some salient features of innate programing and memory, patho-physiological consequences, underlying mechanisms, and current pressing issues.
\end{abstract}

innate immunity, memory, dynamics, priming, tolerance, exhaustion, resolving and non-resolving inflammation

Citation: Yuan, R., and Li, L. (2016). Dynamic modulation of innate immunity programming and memory. Sci China Life Sci 59, 38-43. doi: $10.1007 / \mathrm{s} 11427-015-4998-\mathrm{x}$

\section{INTRODUCTION}

Mediated by innate pattern recognition-receptors, innate immune cells may respond to broad molecular patterns and are responsible for the modulation of general immune environments, through the expression of diverse inflammatory mediators. Unlike the adaptive immune cells that are known to adopt distinct memory states toward highly specific antigens, innate immune cells were thought to be highly plastic and have no memory of prior challenges. To the contrary, data from recent studies increasingly suggest that innate immune cells may also be able to adopt "rudimentary" memory states under distinct challenge conditions. For example, some of the earlier studies suggest that unique combinations of cytokine environments may program several distinct macrophage phenotypes such as the pro-inflammatory M1, the anti-inflammatory M2, and various intermediate subsets (Gordon, 2007; Martinez et al., 2008). Innate macrophages and monocytes may also adopt highly dynam-

*Corresponding author (email: 1wli@vt.edu) ic "primed" or "tolerant" state that may correlate with the establishment of "non-resolving" vs. "resolving" inflammation depending on the signal strength and history of prior challenges (Morris et al., 2014; Morris, 2015). Clinical observations support the existence of distinct innate leukocyte subsets in vivo, and render the pathophysiological significance of innate programming and memory. The nonresolving pro-inflammatory macrophages are often correlated with the pathogenesis of inflammatory diseases, and the resolving anti-inflammatory macrophages tend to associate with tissue repair (Mosser and Edwards, 2008). Here we review some of the key features and underlying mechanisms of innate leukocyte programming.

\section{DISTINCT PROGRAMMING BY DIFFERENT CYTOKINE ENVIRONMENTS}

Innate leukocytes such as macrophages have the potential to express a large array of cellular mediators including cytokines, chemokines, co-stimulatory molecules, and lipid mediators. However, depending upon the nature of the external 
stimulants, the gene expression profiles of macrophages can vary dramatically. Macrophages treated with interferon gamma (IFN $\gamma$ ) are skewed to selectively express proinflammatory mediators as represented by IL-12 or tumor necrosis factor $\alpha$ (TNF $\alpha$ ), and fail to express antiinflammatory mediators such as IL-10 or TGF $\beta$. IFN $\gamma$ - conditioned macrophages are named as M1 macrophages (Gordon and Taylor, 2005). In contrast, the gene expression profiles of macrophages treated with IL-4 are drastically different, and are represented by the selective expression of anti-inflammatory mediators such as arginase 1 (Gordon and Taylor, 2005). IL-4 pre-conditioned macrophages are also classified as the M2 macrophages. On the other hand, immune complexes coupled with lipopolysaccharide (LPS) may program macrophages into various M2b subsets with selective expression of cell-surface co-stimulatory molecules (Mosser and Edwards, 2008). TGF $\beta$ or IL-10-treated macrophages tend to adopt a suppressive phenotype with selective expression of IL-10 (Table 1).

In terms of molecular mechanisms, epigenetic programming through IFN-activated STAT1 may be responsible for the global histone acetylation associated with the inflammatory gene loci (Qiao et al., 2013). Likewise, IL-4 or TGF $\beta$ may induce distinct epigenetic programming events in macrophages, leading to the differential skewing of gene expression profiles (Lawrence and Natoli, 2011).

\section{COMBINATORIAL PROGRAMMING WITH MULTIPLE IMMUNE STIMULANTS}

Although well-defined and distinct culturing stimulus may offer clear differentiation phenotypes of macrophages/monocytes, it may not reflect the complex in vivo environment where monocytes may constantly face changing landscapes of multiple cellular mediators in a temporal and

Table 1 Programming dynamics of innate monocytes/macrophages ${ }^{\text {a) }}$

\begin{tabular}{|c|c|c|}
\hline Conditions & Inducers & Effects \\
\hline \multirow[t]{4}{*}{$\begin{array}{l}\text { Programming through } \\
\text { different challenges }\end{array}$} & IFN $\gamma$ & $\begin{array}{l}\text { M1 macrophage/ } \\
\text { monocyte }\end{array}$ \\
\hline & IL-4, IL-13 & $\begin{array}{l}\text { M2a macrophage/ } \\
\text { monocyte }\end{array}$ \\
\hline & ICs+LPS & $\begin{array}{l}\text { M2b macrophage/ } \\
\text { monocyte }\end{array}$ \\
\hline & TGF $\beta$, IL-10 & $\begin{array}{l}\text { M2c macrophage/ } \\
\text { monocyte }\end{array}$ \\
\hline \multirow{2}{*}{$\begin{array}{l}\text { Sequential/ } \\
\text { combinational } \\
\text { programming }\end{array}$} & $\beta$-glucan+LPS & $\begin{array}{l}\text { Selective training of } \\
\text { inflammatory monocyte }\end{array}$ \\
\hline & IFN $\gamma+\mathrm{LPS}$ & $\begin{array}{l}\text { Selective priming of } \\
\text { inflammatory monocyte }\end{array}$ \\
\hline \multirow[t]{2}{*}{$\begin{array}{l}\text { Signal-strength depend- } \\
\text { ent programming }\end{array}$} & $\begin{array}{l}\text { Super low dose } \\
\text { LPS }\end{array}$ & $\begin{array}{l}\text { Non-resolving low-grade } \\
\text { inflammatory monocyte } \\
\left(\mathrm{M}_{\mathrm{L}}\right)\end{array}$ \\
\hline & Higher dose LPS & $\begin{array}{l}\text { Transient inflammatory } \\
\text { and resolving tolerant } \\
\text { monocyte }\end{array}$ \\
\hline
\end{tabular}

a) ICs, Immune complexes. spatial fashion. To address whether monocytes may gain potential "memory" from previous challenges, experimental systems were designed to provide simultaneous or sequential challenges to monocytes with multiple distinct stimulants. For example, monocytes with a prior challenge of beta-glucan were "trained" to respond with a more robust inflammatory response to a subsequent LPS challenge (Netea et al., 2011; Quintin et al., 2012). We reported that retinoic acid may synergize with IL-4 in triggering the expression of M2-type cellular mediators such as arginase 1, while co-stimulation of monocytes with IL-4/retinoic acid with LPS annihilate the expression of arginase 1 (Surace and $\mathrm{Li}, 2013$ ).

These phenomenological studies suggest that intracellular pathways triggered by distinct innate stimulants may converge into inter-twined circuits, and responsible for the cross-talk within monocytes toward multiple stimulants. For example, we and others demonstrated that nuclear receptors (e.g. RAR $\alpha, \mathrm{ROR} \alpha$ ) activated by retinoic acid can not only synergize with signal transducer and activator of transcription 4 (STAT4) activated by IL-4, but also can potently suppress the inflammatory NFKB pathway activated by LPS (Huang and Glass, 2010; Maitra et al., 2011).

Recent mechanistic studies also suggest that the occurrence of the "trained immunity" due to the sequential challenges of distinct stimulants may be the consequence of complex alterations at multiple levels such as metabolic changes as well as epigenetic modification of chromatin structures (Saeed et al., 2014; Cheng et al., 2014). For example, beta-glucan "trained" human monocytes may undergo metabolic changes in favor of aerobic glycolysis that subsequently causes epigenetic re-programming of selected down-stream inflammatory genes (Cheng et al., 2014). Likewise, interferon-gamma mediated priming of macrophage inflammatory responses may also be due to alteration in metabolic pathways, down-regulation of PI3K and mTORC1, as well as epigenetic modification of target inflammatory genes (Su et al., 2015).

\section{SIGNAL STRENGTH-DEPENDENT PROGRAMMING OF INNATE LEUKOCYTES}

Recent studies from our group and other suggest another intriguing aspect of innate immunity programming and memory, in that innate leukocytes may not only be able to recognize different combinations of extra-cellular stimulants, but also discern their relative signal strength (Baker et al., 2014; Deng et al., 2013; Lu et al., 2015; Maitra et al., 2012; Maitra et al., 2011; Morris et al., 2014). This is reflected in the cardinal example of endotoxin priming and tolerance. A salient example of selective innate preconditioning is the paradigm of endotoxin priming and tolerance (Morris and Li, 2012). Host monocytes/macrophages are the most potent responders to bacterial endotoxin, LPS (Gordon, 2007). Most studies regarding cellular responses 
to LPS utilized higher tolerant dosages of LPS (although often referred as "low dose" in the literature) $\left(>10 \mathrm{ng} \mathrm{mL}^{-1}\right.$ in vitro culture, $>1 \mu \mathrm{g}$ mouse $\mathrm{e}^{-1}$ or $>50 \mu \mathrm{g} \mathrm{kg}^{-1}$ body weight in vivo injection). Higher doses LPS cause robust induction of pro-inflammatory mediators in monocytes/macrophages through the Toll-Like-Receptor 4 (TLR4) pathway (Kawai and Akira, 2007). Shortly after the initial wave of expression, host macrophages develop a state of "endotoxin tolerance", in which the expressions of pro-inflammatory mediators are suppressed (Henricson et al., 1993; Li et al., 2000; West and Heagy, 2002). Endotoxin tolerance serves as a compensatory mechanism for the resolution of inflammation (Jacinto et al., 2002; Medvedev et al., 2002). In stark contrast, subclinical super-low levels of circulating LPS $\left(\sim 1-100 \mathrm{pg} \mathrm{mL}^{-1}\right)$ in experimental animals and humans with adverse health conditions do not induce compensatory tolerance (Hirohashi and Morrison, 1996; Li et al., 2000; Sturm, 2002; Zhang and Morrison, 1993). Instead, we and others have shown that super-low dose LPS $(\sim 1-100$ pg mL $\mathrm{m}^{-1}$ in vitro, $100 \mathrm{pg}-10 \mathrm{ng}$ mouse $\mathrm{s}^{-1}$ or $5 \mathrm{ng} \mathrm{kg}^{-1}-0.5 \mu \mathrm{g}$ $\mathrm{kg}^{-1}$ body weight in vivo injection) "primes" macrophages for a more robust response to a secondary LPS challenge in vitro and in vivo, a phenomenon known as the "Shwartzman reaction" (Deng et al., 2013; Henricson et al., 1993; Hirohashi and Morrison, 1996; Zhang and Morrison, 1993) (Figure 1).

One of the critical deficiencies of existing studies regarding priming and tolerance is the short time course and limited exposure of LPS. Almost all studies employ a single LPS challenge to induce priming or tolerance within a $24 \mathrm{~h}$ time period. We recently tested the hypothesis that repeated challenges with super-low dose LPS for a longer period of time may polarize monocytes into a low-grade inflammatory state. We observed that repeated challenges with super-low dose LPS indeed establishes a low-grade inflammatory monocyte phenotype $\left(\mathrm{M}_{\mathrm{L}}\right)$ in both murine and hu-

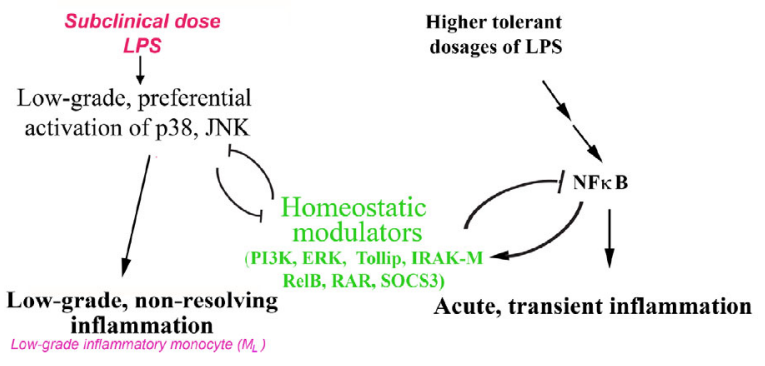

Figure 1 (color online) A schematic diagram illustrating the dynamic circuitry potentially responsible for the establishment of either low-grade non-resolving inflammation or acute resolving inflammation. For the establishment of acute resolving inflammation, the occurrence of negative feedbacks due to the activation of homeostatic modulators such as PI3K/AKT, IRAK-M, Tollip, SOCS3, and ERK would be required. On the other hand, the clearance of these negative homeostatic modulators would favor the low-grade non-resolving inflammation and the establishment of a low-grade inflammatory monocyte state $\left(\mathrm{M}_{\mathrm{L}}\right)$. man primary monocyte (Yuan et al., 2016).

Through toll-like-receptor 4 (TLR4), Higher dosages of LPS induce transient and resolving inflammation through the activation of both classical and alternative NFKB pathways (O’Neill, 2002; Yoza et al., 2006) The classical NFkB pathway contributes to the robust yet transient induction of pro-inflammatory mediators (Kawai and Akira, 2007). As a compensatory mechanism, higher dose LPS also activates the anti-inflammatory PI3K pathway that leads to the activation and induction of several negative regulators such as MKP-1, RelB, pAKT, pERK, ROR $\alpha$, and RAR $\alpha$, which serve as negative regulators of pro-inflammatory $\mathrm{NF \kappa B}$ function (Chan et al., 2005; Chen et al., 2009a; Chen et al., 2009b; Li et al., 2009; Piao et al., 2009; Yoza et al., 2006). This underlies the phenotype of "endotoxin tolerance" (Yoza et al., 2006).

In stark contrast, the mechanisms for endotoxin priming are less well studied and poorly understood. We first reported that super-low dose LPS fails to induce negative regulators such as nuclear receptors (RAR $\alpha, \mathrm{ROR} \alpha$, etc), PI3K/Akt pathway, ERK, Tollip and RelB in innate leukocytes (Maitra et al., 2011) (Maitra et al., 2012). This may explain the lack of tolerance by super-low dose LPS. Instead, we observed that super-low dose LPS suppresses aforementioned negative regulators and primes monocytes/ macrophages for more robust expression of pro-inflammatory mediators. Among intracellular components of TLR4 pathway, we reported that IRAK-1 is critical for the effect of super-low dose LPS and priming of innate leukocytes (Maitra et al., 2012; Maitra et al., 2011). Mechanistically, we documented that IRAK-1 is required for suppressor clearance induced by super-low dose LPS (Maitra et al., 2012). In contrast, higher dose LPS activates PI3K and leads to IRAK-1 degradation (Li et al., 2000; Noubir et al., 2004) (Figure 1).

These studies may explain many previous clinical observations and bear far-reaching pathophysiological consequences. For example, varying dosages of endotoxin have been shown to differentially affect the pathogenesis of asthma, atherosclerosis, and sepsis in animal studies (Chen et al., 2015; Eisenbarth et al., 2002; Kallio et al., 2008; Maitra et al., 2012; Wiesner et al., 2010). Differential development of asthma and other inflammatory diseases associated with people from developing countries prompted the "hygiene theory" that resonates with these dynamic programming of innate leukocytes (Vandenbulcke et al., 2006). It may be an evolutionary survival advantage for human hosts to adopt a compensatory homeostatic tolerance when challenged with higher dosages of innate stimulants. On the other hand, chronic exposure to minute amount of innate stimulants may dispense the tonic homeostatic mechanism of tolerance, and develop low-grade wax and wane inflammation. As the chronic inflammation persists, innate leukocytes as well as their hematopoietic precursors may be exhausted, which may contribute to immune exhaustion and 
"inflammaging" during the aging process (Shaw et al., 2013).

The dynamic responses to varying dosages of innate stimulants are not limited to endotoxin, and are observed with other agonists such as TLR7/8 agonists (Christensen et al., 2006; Gorden et al., 2005). This may be a generalized phenomenon in innate leukocytes during their dynamic and tailored adaption to changing environments over time.

\section{SYSTEMS BIOLOGY OF INNATE PROGRAMING AND MEMORY}

The highly complex and diverse profiles of leukocyte programming pose immense challenges for experimental characterization. Recent advances in computational systems analyses may offer significant assistance in deciphering complex leukocyte dynamics and memory. In collaboration with computational biologists, we have identified several salient functional motifs required for the establishment of priming and homeostatic tolerant states in innate leukocytes. For the homeostatic tolerance, the sustained presence of robust homeostatic negative regulator(s) would be required (Fu et al., 2012; Su J, 2009). This is consistent with experimental observations from our lab and others that reveal the induction of multiple negative regulators during the homeostatic tolerance of innate leukocytes such as Tollip, PI3K, ERK, IRAK-M, SOCS3, and I $\mathrm{BB} \alpha$ (Chan et al., 2005; Maitra et al., 2012; Piao et al., 2009; Yoza et al., 2006). These homeostatic modulators may function at multiple levels during the signaling process. On the other hand, for the priming process to occur, the pathway switching from the induction to the clearance and disruption of negative suppressors must occur. Our laboratory has provided data confirming the clearance of suppressors such as Tollip and IRAK-M, as well as suppression of ERK and PI3K during the priming process of innate leukocytes (Baker et al., 2014; Deng et al., 2013; Maitra et al., 2012; Maitra and Li, 2013).

Computational and experimental analyses also converge to suggest a dynamic circuit consisting of two mutually-inhibitory arms of positive and negative regulators, which may give rise to the bi-stable states of cellular priming and homeostatic tolerance (Fu et al., 2012). This salient motif may be critically involved in the sensing of rising signal strength of innate stimulants, and responsible for the fine-tuning of leukocyte functions. Intriguingly, systems analyses suggest that this motif may also be at work during the dynamic differentiation of $\mathrm{T}$ helper cells into distinct Th1/Th2/Th17/Treg states (Hong et al., 2012; Hong, 2011), and may hold general significance in the modulation immune cells.

The distinct programming of innate leukocytes may also be finely modulated by spatial coordination of signaling circuitries. For example, we observed that the Tollip molecule may serve dual roles as both a positive and a negative regulator during the activation process of innate leukocytes, depending upon its sub-cellular localization. Lysosomal Tollip is crucial for maintaining homeostasis by facilitating the fusion of lysosome and autophagosome, and the completion of autophagy (Baker et al., 2015). However, unknown modification may drive its translocation away from lysosome to mitochondria, and switch Tollip from a homeostatic factor to a stress-promoting molecule (Baker et al., 2015; Maitra et al., 2012).

\section{INNATE LEUKOCYTE MEMORY IN RESOLVING AND NON-RESOLVING INFLAMMATION}

Dynamic modulation and memory of innate immunity may have expanding roles in the pathogenesis and resolution of inflammatory diseases ranging from acute sepsis to chronic diseases such as atherosclerosis, diabetes, impaired wound healing, neurological disease and aging. During acute inflammatory disease such as acute injury, trauma, acute colitis and sepsis, dysregulated innate immune programming may lead to defective host defense toward invasive microbes, as well as dysregulated tissue inflammation. We recently reported that pre-conditioning with super-low dose endotoxin pre-disposes animals for increased mortality in subsequence sepsis (Chen et al., 2015). Mice preconditioned with subclinical super-low level of endotoxin exhibit severe tissue damage, inflammation, increased bacterial load in circulation, and elevated mortality when subjected to cecal-ligation and puncture (CLP). This is in contrast to the protective phenomenon with CLP mice pre-conditioned with higher tolerant level of LPS. Tolerant dosages of LPS skews an anti-inflammatory phenotype in both monocyte and neutrophils as reflected in reduced expression of pro-inflammatory mediators (Chen et al., 2015). In contrast, super-low dose LPS polarizes a low-grade pro-inflammatory state in monocytes and neutrophils, conducive of tissue damage (Chen et al., 2015). Furthermore, we demonstrated that varying dosages of LPS differentially modulate the formation of neutrophil extracellular trap (NET) in neutrophils. Super-low dose LPS suppresses the homeostatic circuits such as PI3K/AKT and ERK that are required for the generation of neutrophil NET (Chen et al., 2015; Kopanakis et al., 2013). In contrast, higher tolerant dosages of LPS activates PI3K/ERK and facilitate NET formation (Kopanakis et al., 2013). Balancing homeostasis in innate leukocytes may hold potential in treating acute injury and sepsis. During the chronic inflammatory processes, our recent study suggest that polarization of the low-grade inflammatory state in innate leukocytes through the disruption of homeostatic pathways may similarly play a key role in sustaining the non-resolving inflammation that underlies the pathogenesis of atherosclerosis, diabetes, delayed wound repair, and ageing (Yuan et al., 2016). We demonstrated that persistent challenges with super-low dose LPS may polarize monocytes into a novel low-grade in- 
flammatory state $\left(\mathrm{M}_{\mathrm{L}}\right)$ that is conducive for chronic non-resolving inflammation (Yuan et al., 2016).

Thus, homeostatic resolution of innate leukocyte activation through the induction of homeostatic molecules, autophagy, and biogenesis of homeostatic organelles such as lysosomes may be a viable approach for the treatment of both acute and chronic inflammatory disease. To this regard, potential promising compounds such as Tauroursodeoxycholic Acid (TUDCA) and trehalose have been tested and shown to have beneficial effects in maintaining partial homeostasis in innate leukocytes and the treatment of inflammatory and infectious diseases (Chen et al., 2015; Martinez-Lopez et al., 2013; Wu et al., 2015).

\section{CONCLUSION}

In summary, we are witnessing the emerging concept of "innate memory", and what is known so far only may reflect a tip of an iceberg in terms of its complexity and translational potential. The continued integration of experimental and computational approaches, coupled with genomics and proteomics analyses of large datasets, may yield rich and exciting profiles of innate leukocyte activation. With particular urgency, epigenetic analyses should be performed to define key molecular markers that may assist the classification and characterization of novel innate leukocyte subsets in health and disease. Translational studies in humans will help to assign clinical relevance to these emerging "memory" innate leukocytes in the future.

Compliance and ethics The author(s) declare that they have no conflict of interest.

Acknowledgements We appreciate the discussion of the Li lab, and the support from the National Institute of Health grant HL 115835.

Baker, B., Geng, S., Chen, K., Diao, N., Yuan, R., Xu, X., Dougherty, S., Stephenson, C., Xiong, H., Chu, H.W., and Li, L. (2015). Alteration of lysosome fusion and low-grade inflammation mediated by super-low-dose endotoxin. J Biol Chem 290, 6670-6678.

Baker, B., Maitra, U., Geng, S., and Li, L. (2014). Molecular and cellular mechanisms responsible for cellular stress and low-grade inflammation induced by super-low dose endotoxin. J Biol Chem 289, 16262-16269.

Chan, C., Li, L., McCall, C.E., and Yoza, B.K. (2005). Endotoxin tolerance disrupts chromatin remodeling and NF-kappaB transactivation at the IL-1beta promoter. J Immunol 175, 461-468.

Chen, K., Geng, S., Yuan, R., Diao, N., Upchurch, Z., and Li, L. (2015). Super-low dose endotoxin pre-conditioning exacerbates sepsis mortality. EBioMedicine 2, 324-333.

Chen, X., El Gazzar, M., Yoza, B.K., and McCall, C.E. (2009a). The NF-kappaB factor RelB and histone H3 lysine methyltransferase G9a directly interact to generate epigenetic silencing in endotoxin tolerance. J Biol Chem 284, 27857-27865.

Chen, X., Yoza, B.K., El Gazzar, M., Hu, J.Y., Cousart, S.L., and McCall, C.E. (2009b). RelB sustains IkappaBalpha expression during endotoxin tolerance. Clin Vaccine Immunol 16, 104-110.

Cheng, S.C., Quintin, J., Cramer, R.A., Shepardson, K.M., Saeed, S., Kumar, V., Giamarellos-Bourboulis, E.J., Martens, J.H., Rao, N.A.,
Aghajanirefah, A., Manjeri, G.R., Li, Y., Ifrim, D.C., Arts, R.J., van der Veer, B.M., Deen, P.M., Logie, C., O’Neill, L.A., Willems, P., van de Veerdonk, F.L., van der Meer, J.W., Ng, A., Joosten, L.A., Wijmenga, C., Stunnenberg, H.G., Xavier, R.J., and Netea, M.G. (2014). mTORand HIF- $1 \alpha$-mediated aerobic glycolysis as metabolic basis for trained immunity. Science 345, 1250684.

Christensen, S.R., Shupe, J., Nickerson, K., Kashgarian, M., Flavell, R.A., and Shlomchik, M.J. (2006). Toll-like receptor 7 and TLR9 dictate autoantibody specificity and have opposing inflammatory and regulatory roles in a murine model of lupus. Immunity 25, 417-428.

Deng, H., Maitra, U., Morris, M., and Li, L. (2013). Molecular mechanism responsible for the priming of macrophage activation. J Biol Chem 288 , 3897-3906.

Eisenbarth, S.C., Piggott, D.A., Huleatt, J.W., Visintin, I., Herrick, C.A., and Bottomly, K. (2002). Lipopolysaccharide-enhanced, toll-like receptor 4-dependent $\mathrm{T}$ helper cell type 2 responses to inhaled antigen. $\mathrm{J}$ Exp Med 196, 1645-1651.

Fu, Y., Glaros, T., Zhu, M., Wang, P., Wu, Z., Tyson, J.J., Li, L., and Xing, J. (2012). Network topologies and dynamics leading to endotoxin tolerance and priming in innate immune cells. PLoS Comput Biol 8, e1002526.

Gorden, K.B., Gorski, K.S., Gibson, S.J., Kedl, R.M., Kieper, W.C., Qiu, X., Tomai, M.A., Alkan, S.S., and Vasilakos, J.P. (2005). Synthetic TLR agonists reveal functional differences between human TLR7 and TLR8. J Immunol 174, 1259-1268.

Gordon, S. (2007). The macrophage: past, present and future. Eur J Immunol 37 Suppl 1, S9-S17.

Gordon, S., and Taylor, P.R. (2005). Monocyte and macrophage heterogeneity. Nat Rev Immunol 5, 953-964.

Henricson, B.E., Manthey, C.L., Perera, P.Y., Hamilton, T.A., and Vogel, S.N. (1993). Dissociation of lipopolysaccharide (LPS)-inducible gene expression in murine macrophages pretreated with smooth LPS versus monophosphoryl lipid A. Infect Immun 61, 2325-2333.

Hirohashi, N., and Morrison, D.C. (1996). Low-dose lipopolysaccharide (LPS) pretreatment of mouse macrophages modulates LPS-dependent interleukin-6 production in vitro. Infect Immun 64, 1011-1015.

Hong, T., Xing, J., Li, L., and Tyson, J.J. (2012). A simple theoretical framework for understanding heterogeneous differentiation of CD4+ T cells. BMC Syst Biol 6, 66.

Hong, T.X., J.; Li, L.; Tyson, J. (2011). A mathematical model for the reciprocal differentiation of $\mathrm{T}$ helper 17 cells and induced regulatory $\mathrm{T}$ cells. PLoS Comput Biol 7, e1002122.

Huang, W., and Glass, C.K. (2010). Nuclear receptors and inflammation control: molecular mechanisms and pathophysiological relevance. Arterioscler Thromb Vasc Biol 30, 1542-1549.

Jacinto, R., Hartung, T., McCall, C., and Li, L. (2002). Lipopolysaccharide- and lipoteichoic acid-induced tolerance and cross-tolerance: distinct alterations in IL-1 receptor-associated kinase. J Immunol 168, 6136-6141.

Kallio, K.A., Buhlin, K., Jauhiainen, M., Keva, R., Tuomainen, A.M., Klinge, B., Gustafsson, A., and Pussinen, P.J. (2008). Lipopolysaccharide associates with pro-atherogenic lipoproteins in periodontitis patients. Innate Immun 14, 247-253.

Kawai, T., and Akira, S. (2007). TLR signaling. Semin Immunol 19, 24-32.

Kopanakis, K., Tzepi, I.M., Pistiki, A., Carrer, D.P., Netea, M.G., Georgitsi, M., Lymperi, M., Droggiti, D.I., Liakakos, T., Machairas, A., and Giamarellos-Bourboulis, E.J. (2013). Pre-treatment with low-dose endotoxin prolongs survival from experimental lethal endotoxic shock: benefit for lethal peritonitis by Escherichia coli. Cytokine 62, 382-388.

Lawrence, T., and Natoli, G. (2011). Transcriptional regulation of macrophage polarization: enabling diversity with identity. Nat Rev Immunol $11,750-761$.

Li, L., Chen, S.F., and Liu, Y. (2009). MAP kinase phosphatase-1, a critical negative regulator of the innate immune response. Int $\mathrm{J}$ Clin Exp Med 2, 48-67.

Li, L., Cousart, S., Hu, J., and McCall, C.E. (2000). Characterization of interleukin-1 receptor-associated kinase in normal and endotox- 
in-tolerant cells. J Biol Chem 275, 23340-23345.

Lu, G., Zhang, R., Geng, S., Peng, L., Jayaraman, P., Chen, C., Xu, F., Yang, J., Li, Q., Zheng, H., Shen, K., Wang, J., Liu, X., Wang, W., Zheng, Z., Qi, C.F., Si, C., He, J.C., Liu, K., Lira, S.A., Sikora, A.G., Li, L., and Xiong, H. (2015). Myeloid cell-derived inducible nitric oxide synthase suppresses M1 macrophage polarization. Nat Commun 6, 6676

Maitra, U., Deng, H., Glaros, T., Baker, B., Capelluto, D.G., Li, Z., and Li, L. (2012). Molecular mechanisms responsible for the selective and low-grade induction of proinflammatory mediators in murine macrophages by lipopolysaccharide. J Immunol 189, 1014-1023.

Maitra, U., Gan, L., Chang, S., and Li, L. (2011). Low-dose endotoxin induces inflammation by selectively removing nuclear receptors and activating CCAAT/enhancer-binding protein \{delta\}. J Immunol 186, 4467-4473.

Maitra, U., and Li, L. (2013). Molecular mechanisms responsible for the reduced expression of cholesterol transporters from macrophages by low-dose endotoxin. Arterioscler Thromb Vasc Biol 33, 24-33.

Martinez-Lopez, N., Athonvarangkul, D., Mishall, P., Sahu, S., and Singh, R. (2013). Autophagy proteins regulate ERK phosphorylation. Nat commun 4, 2799.

Martinez, F.O., Sica, A., Mantovani, A., and Locati, M. (2008). Macrophage activation and polarization. Front Biosci 13, 453-461.

Medvedev, A.E., Lentschat, A., Wahl, L.M., Golenbock, D.T., and Vogel, S.N. (2002). Dysregulation of LPS-induced Toll-like receptor 4-MyD88 complex formation and IL-1 receptor-associated kinase 1 activation in endotoxin-tolerant cells. J Immunol 169, 5209-5216.

Morris, M., Gilliam, E., Button, J., and Li, L. (2014). Dynamic modulation of innate immune response by varying dosages of LPS in human monocytic cells. J Bio Chem 289, 21584-21590.

Morris, M., and Li, L. (2012). Molecular mechanisms and pathological consequences of endotoxin tolerance and priming. Arch Immunol Ther Exp (Warsz) 60, 13-18.

Morris, M.G.E., Li, L. (2015). Innate immune programming by endotoxin and its pathological consequences. Front Immunol 5, 680 1-8.

Mosser, D.M., and Edwards, J.P. (2008). Exploring the full spectrum of macrophage activation. Nat Rev Immunol 8, 958-969.

Netea, M.G., Quintin, J., and van der Meer, J.W. (2011). Trained immunity: a memory for innate host defense. Cell Host Microbe 9, 355-361.

Noubir, S., Hmama, Z., and Reiner, N.E. (2004). Dual receptors and distinct pathways mediate interleukin-1 receptor-associated kinase degradation in response to lipopolysaccharide. Involvement of CD14/TLR4, CR3, and phosphatidylinositol 3-kinase. J Biol Chem 279, 25189-25195.

O'Neill, L.A. (2002). Signal transduction pathways activated by the IL-1 receptor/toll-like receptor superfamily. Curr Top Microbiol Immunol 270, 47-61.

Piao, W., Song, C., Chen, H., Diaz, M.A., Wahl, L.M., Fitzgerald, K.A., Li, L., and Medvedev, A.E. (2009). Endotoxin tolerance dysregulates MyD88- and Toll/IL-1R domain-containing adapter inducing IFN-beta-dependent pathways and increases expression of negative regulators of TLR signaling. J Leukocyte Biol 86, 863-875.

Qiao, Y., Giannopoulou, E.G., Chan, C.H., Park, S.H., Gong, S., Chen, J., $\mathrm{Hu}$, X., Elemento, O., and Ivashkiv, L.B. (2013). Synergistic activation of inflammatory cytokine genes by interferon-gamma-induced chroma- tin remodeling and toll-like receptor signaling. Immunity 39, 454-469.

Quintin, J., Saeed, S., Martens, J.H., Giamarellos-Bourboulis, E.J., Ifrim, D.C., Logie, C., Jacobs, L., Jansen, T., Kullberg, B.J., Wijmenga, C., Joosten, L.A., Xavier, R.J., van der Meer, J.W., Stunnenberg, H.G., and Netea, M.G. (2012). Candida albicans infection affords protection against reinfection via functional reprogramming of monocytes. Cell Host Microbe 12, 223-232.

Saeed, S., Quintin, J., Kerstens, H.H., Rao, N.A., Aghajanirefah, A., Matarese, F., Cheng, S.C., Ratter, J., Berentsen, K., van der Ent, M.A., Sharifi, N., Janssen-Megens, E.M., Ter Huurne, M., Mandoli, A., van Schaik, T., Ng, A., Burden, F., Downes, K., Frontini, M., Kumar, V., Giamarellos-Bourboulis, E.J., Ouwehand, W.H., van der Meer, J.W., Joosten, L.A., Wijmenga, C., Martens, J.H., Xavier, R.J., Logie, C., Netea, M.G., and Stunnenberg, H.G. (2014). Epigenetic programming of monocyte-to-macrophage differentiation and trained innate immunity. Science 345, 1251086.

Shaw, A.C., Goldstein, D.R., and Montgomery, R.R. (2013). Age-dependent dysregulation of innate immunity. Nat Rev Immunol $13,875-887$.

Sturm, R. (2002). The effects of obesity, smoking, and drinking on medical problems and costs. Health Aff (Millwood) 21, 245-253.

Su J, Z.T., Tyson J, and Li L (2009). The interleukin-1 receptor-associated kinase M (IRAK-M) selectively inhibits the alternative, instead of the classical NFאB pathway. J Innate Immun, 164-174.

Su, X., Yu, Y., Zhong, Y., Giannopoulou, E.G., Hu, X., Liu, H., Cross, J.R., Rätsch, G., Rice, C.M., and Ivashkiv, L.B. (2015). Interferon- $\gamma$ regulates cellular metabolism and mRNA translation to potentiate macrophage activation. Nat Immunol 16, 838-849.

Surace, M.J., and Li, L. (2013). Potent suppression of arginase 1 expression in murine macrophages by low dose endotoxin. Am J Clin Exp Immunol 2, 117-123.

Vandenbulcke, L., Bachert, C., Van Cauwenberge, P., and Claeys, S. (2006). The innate immune system and its role in allergic disorders. Int Arch Allergy Immunol 139, 159-165.

West, M.A., and Heagy, W. (2002). Endotoxin tolerance: a review. Crit Care Med 30, S64-S73.

Wiesner, P., Choi, S.H., Almazan, F., Benner, C., Huang, W., Diehl, C.J., Gonen, A., Butler, S., Witztum, J.L., Glass, C.K., and Miller, Y.I. (2010). Low doses of lipopolysaccharide and minimally oxidized low-density lipoprotein cooperatively activate macrophages via nuclear factor kappab and activator protein-1: possible mechanism for acceleration of atherosclerosis by subclinical endotoxemia. Circulat Res 107, $56-65$.

Wu, Q., Jiang, D., Huang, C., van Dyk, L.F., Li, L., and Chu, H.W. (2015). Trehalose-mediated autophagy impairs the anti-viral function of human primary airway epithelial cells. PLoS One 10, e0124524.

Yoza, B.K., Hu, J.Y., Cousart, S.L., Forrest, L.M., and McCall, C.E. (2006). Induction of RelB participates in endotoxin tolerance. J Immunol 177, 4080-4085.

Yuan, J., Geng, S., Chen, K., Diao, N., Chu, H.W., and Li, L. (2016). Low-grade inflammatory polarization of monocytes impairs wound healing. J Pathol, in press.

Zhang, X., and Morrison, D.C. (1993). Lipopolysaccharide-induced selective priming effects on tumor necrosis factor alpha and nitric oxide production in mouse peritoneal macrophages. J Exp Med 177, 511-516.

Open Access This article is distributed under the terms of the Creative Commons Attribution License which permits any use, distribution, and reproduction in any medium, provided the original author(s) and source are credited. 